Mittheilung aus der Universitäts-Frauenklinik zu Göttingen.

\title{
Zwei bemerkenswerthe Fälle von Ileus vor und nach der Laparatomie, beide mit Ausgang in Heilung.
}

Von

Dr. R. C a r i o,

r. Assistenzarzt.

Fall I. Linksseitiger Ovarialtumor. Ileus. Laparatomie. Stieldrehung mit Eindrehung des Dünndarmes. Heilung.

Frau H., Arbeitersfrau aus Negenborn, 38 Jahre alt, fand Aufnahme in der Klinik am 17. December 1889. Sie hat seit einem Jahre eine Auftreibung des Leibes bemerkt, welche nach und nach stärker geworden ist, auch sollen häufige Schmerzen im Rücken und Leibe aufgetreten sein. Hin und wieder will sie an Verstopfung und Drang zum Urinlassen gelitten haben.

Befund: Grosse und magere Frau, deren verfallene Gesichtszüge und ergrauendes Haar sie älter erscheinen lassen, als sie ist. Der Leib ist aufgetrieben, der Nabel fast verstrichen, die Bauchhaut glänzend. Leibesumfang $104 \mathrm{~cm}$. Die Palpation ergiebt einen Tumor, der bis dicht oberhalb des Nabels reicht, sich seitlich gut abgrenzen lässt und gedämpften Percussionsschall zeigt. Bei der inneren Untersuchung fühlt man durch das vordere Scheidengewölbe denselben Tumor, der links strangförmig mit dem Uterus in Verbindung. zu stehen scheint. Der nicht vergrösserte Uterus hinter dem Tumor. Nach dem Herabziehen des Uterus fühlt man rom Mastdarme aus deutlich, dass der Tumor mit der linken Seite des Uterus durch einen Strang in Verbindung steht. Diag nose: Linksseitiger gestielter Ovarialtumor. 
Die Ovariotomie musste mehrmals verschoben werden, weil gerade die Influenza in der Anstalt hauste, und konnte erst nach Neujahr ausgeführt werden.

Am 5. Januar hatte die Kranke Morgens einen Esslöffel voll Oleum ricini erhalten und war zwei Mal auf dem Aborte gewesen, als sie Nachmittags eine plötzliche Temperatursteigerung a uf 38,3 bei 122 Pulsen bekam, die aber am anderen Morgen auf $37^{\circ}$ und 76 Pulse zurückgegangen war. Man war zur Annahme von Influenza geneigt. Am 7. Januar Nachmittags begannen aber Leibschmerzen, die in der folgendem Nacht stärker wurden, auch trotz $20 \mathrm{gtt}$. Tincturae opii simplicis zunahmen, ohne dass die Temperatur anstieg, während der Puls sich auf 100 hob. Abführmittel ohne Wirkung, auf ein Klysma nur geringe Ausleerung.

Am 8. Januar unerträgliche Schmerzen, hauptsächlich im Epigastrium, anfallsweise, und am Vormittage Erbrechen von gallig gefärbtem Schleime. Athmung nicht gestört und über den Lungen keine Veränderungen nachweisbar. Der Leib erscheint jetzt stärker ausgedehnt als bei der ersten Untersuchung, Umfang $106 \mathrm{~cm}$, Nabel vollständig verstrichen. Um Mittag wird eine Tasse Bouilion sofort wieder erbrochen, ebenso etwas später Ricinusöl. Die Schmerzen am Nachmittage so stark, dass um 21/2 Uhr 0,01 Morphium subcutan und, da nunmehr Verdacht a uf Ileus besteht, $10 \mathrm{gtt}$. Tincturae opii simplicis innerlich gegeben werden. Leib nirgends druckempfindlich, Schmerzen in der Pylorusgegend werden als brennende bezeichnet. Nach zweistündiger Ruhepause Schmerzen allmälig wieder stärker, machen um 6 Uhr eine gleiche Dosis Morphium nöthig. Todesangst und A thembeklemmungen, dabei sieht die Frau erdfahl aus und verfällt zusehends. Puls wird klein, steigt a uf 110 bis 130 , Temperatur bleibt normal. Um $7 \mathbf{1}_{\mathbf{2}}$ Uhr erbricht die Frau etwa 1 Liter erbsengelber, trüber, nach Koth stinkender Flüssigkeit, die angeblich auch nach Koth schmeckt. Da Ileus nun erwiesen ist, Opium in häufigen Dosen, und zwar zunächst um $8^{1} / 4$ Uhr 30 gtt. Tincturae opii simplicis. Nach einer halben Stunde Erbrechen von gleicher Beschaffenheit. Um 91/4 Uhr 15.gtt. Opiumtinctur. Dann wieder heftige, kolikartige Schmerzen. Von jetzt ab kleine Eisstückchen zu schlucken gegeben. Um $10^{1} / 2$ Uhr 15 gtt. Opiumtinctur. Auf ein Klysma von 2 Liter werden wenige, aber harte Kothmassen entleert. Um 11 Uhr Koth- 
brechen, nach einer halben Stunde 15 gtt. Opiumtinctur. Um $12 \mathrm{Uhr}$ leichter warmer Breiumschlag auf die Magengrube, der von Zeit zu Zeit erneuert wird. Zwei weitere grosse Klystiere bis zu 3 Liter auf einmal - wirkten nicht. Um $1 \frac{1}{2}, 5,6 \frac{1}{2}$ und $83 /{ }_{4} \mathrm{Uhr}$ Kothbrechen. In den Zwischenzeiten noch $45 \mathrm{gtt}$. Opiumtinctur in drei Dosen gegeben. Im ganzen wurden mehr als 4 Liter erbrochen mit immer entschiedenerem Kothgeruche.

Die Diagnose musste nun lauten: Ileus, von dem Ovarialtumor durch Stieldrehung oder auf andere Weise hervorgerufen. Die Therapie musste, ohne dass länger Zeit mit Vorbereitungen verloren wurde, in sofortiger Laparatomie bestehen.

Am 9. Januar um $8^{3 / 4}$ Uhr Morgens kurz nach dem letzten Erbrechen Chloroformnarkose. Herr Prof. Runge führt die Operation aus. Der Schnitt in der Linea alba beginnt dicht unter dem Nabel und endet $4 \mathrm{~cm}$ oberhalb der Symphyse. Der Tumor liegt vor und zeigt frische Verwachsungen mit der Bauchwand in den mittleren und unteren Abschnitten. Nach stumpfer Lösung derselben liegt ein Theil des Tumor frei. Punction und Entleerung einer braunrothen, dünnen Flüssigkeit. Der Tumor wird dann an seinem oberen Theile, der keine Verwachsungen zeigt, herausgewälgt und nach leichter Lösung von frischen Verwachsungen an beiden Seiten bis zum Stiele aus der Bauchhöhle herausgezogen. Dabei werden neben ihm sehr stark aufgeblähte und geröthete Dünndarmschlingen sichtbar. Der Stiel zeigt sich gedreht und in die Stieldrehung eine Dünndarmschlinge mit eingedreht. Etwas links seitwärts vom Stiele und von ihm aufwärts hat eine strangförmige, ziemlich frische Verwachsung eine andere Dünndarmschlinge eingeschnürt, jedoch kaum vollständig verschlossen. Beim Aufdrehen des links gewundenen Stieles, der der linken Beckenhälfte angehört, sieht man, dass die verschlossene, eingedrehte Dünndarmschlinge zunächst durch leicht lösbare frische Verwachsungen unter sich und mit dem Stiele verklebt ist. Nach Entfernung dieser Verwachsungen zeigt sich der Stiel noch weiter gedreht und die betroffene Dünndarmschlinge an den Stiel durch eine ältere, feste Verwachsung angeheftet, welche für den Durchgang des Kothes kein Hinderniss bildete. Sie wird von der Stielseite her theils mit dem Messer, theils stumpf gelöst. Der dann völlig aufgedrehte Stiel bildet ein breites Band, welches insofern sehr merkwürdig ist, als es am linken Rande einen hakenförmig nach innen umgeschlagenen Saum besitzt. Die 
Cyste wird nun vollständig entleert, zeigte sich einkammerig und enthielt etwa 5 Liter Flüssigkeit vom specifischen Gewichte 1005, welche nach dem Stehen Blutgerinnsel absetzte. Die vorliegenden Dünndarmschlingen werden nach genauer Säuberung des Operationsfeldes mit den Fingern behutsam verfolgt, und es wird keine weitere Verwachsung, Invagination oder Stenosirung des Darmes entdeckt. Nach der Unterbindung des Stieles durch vier Nähte und tiefer gelegte Gesammtunterbindung wird die Cyste abgetrennt, der Stumpf nochmals durch eine umfassende Naht gesichert, mit 5 proc. Carbolsäure desinficirt und versenkt. Schluss der Bauchwunde durch tiefe, umfassende und oberflächliche Nähte. Die geschlossene Wunde wird jodoformirt, mit einem Gazepolster bedeckt und über eine Wattenschicht eine einfache breite Flanellbinde um den Leib gelegt.

Nach dem Erwachen aus der Narkose haben die kolikartigen Schmerzen aufgehört, es tritt nur der Wundschmerz auf. Am vierten Tage nach der Operation auf Klysmata Stuhlgang. Heilung der Bauchwunde per primam intentionem. Die Frau wird am 6. Februar geheilt und bei völligem Wohlbefinden entlassen.

In der Literatur sind bisher erst neun Fälle bekannt, wo Ileus infolge von Stieldrehung eintrat, und zwar sind diese von Hardy $)$, Ribbentropp ${ }^{2}$ ), Rokitansky ${ }^{3}$, Günther $\mathbf{r}^{4}$ ), Thorn ${ }^{5}$ ), Henry ${ }^{6}$ ) und Hohenegg ${ }^{7}$ ) beschrieben. (Zwei Fälle von $A$ tle $e^{8}$ ) und von $\mathrm{Hande}^{9}$ ) waren mir nicht zugängig.) Nur die Fälle von Thorn, Günther und Hohenegg kamen zur Operation, die beiden letzten mit Ausgang in Heilung, die übrigen wurden auf dem Sectionstische beobachtet.

Hohenegg unterscheidet vier verschiedene Ursachen des Darmverschlusses bei Stieldrehung: 1) Der Ovarialtumor ist bereits nach einer Stieldrehung mit einer Darmschlinge verwachsen und

1) Lancet 1845 , Vol. I, p. 381.

2) Preussische Vereinszeitung 1846.

3) Wiener allgemeine medicinische Zeitschrift 1860, Nr. 4 und Lebrbuch, S. 413.

4) Dissertatio med. Berol. 1879.

5) Dissertatio med. Halens. 1883.

6) American Journal of obstetrics 1880, p. 388 .

7) Wiener klinische Wochenschrift 1888, S. 28.

8) General and differential diaguosis of ovarian tumours, p. 188 (nach Günther a. a. 0 .).

9) Hegar und Kaltenbach, Operative Gynäkologie, S. 249. 
diese wird durch eine neue Drehung abgeknickt (Fall Rokitansky), 2) der lange Stiel des Tumor schlägt sich um eine Darmschlinge herum und schnürt diese ab (Fall Ribbentropp), 3) der Stiel wird durch die Drehung verkürzt und der durch venöse Stase vergrösserte Tumor so auf den Beckeneingang gepresst, dass die Flexura sigmoidea abgeschlossen wird (Fall G ünther), 4) ein Theil des Tumor wird durch die Stieldrehung in das kleine Becken herabgezogen und schliesst hier den Mastdarm ab. Diesen Ursachen ist noch die von Thorn angeführte $(01 \mathrm{~s}$ hausen, Fall 10) hinzuzufügen, wo der Darmverschluss nach Stieldrehung secundär durch nachträgliche Schrumpfung einer Verwachsung eintrat.

In unserem Falle war die Ursache eine ähnliche wie im Falle Rokitansky. Offenbar hatte die Frau schon vor längerer Zeit eine Stieldrehung überstanden, welche als einzige Folge jene ältere Verwachsung einer Darmschlinge mit dem Stiele hinterlassen hatte. Am 5. Januar trat jedenfalls die neue Stieldrehung ein, ohne dass sich ein anderes Zeichen als die einmalige Temperatursteigerung auf $38,3^{\circ}$ zeigte. Beschwerden traten nicht eher auf, als bis sich die Folgen der Eindrehung des Darmes bemerkbar machten, obwohl man, um die frischen Verwachsungen zu erklären, annehmen muss, dass entziundliche Zustände bestanden haben. Sehen wir zunächst von den Ursachen für die Stieldrehung ab und berücksichtigen nur die für den Darmverschluss, so ist es klar, dass in unserem Falle, wo die Darmverwachsung sich am Stiele fand, bei einer neuen Stieldrehung der geringste Widerstand zu überwinden war, um einen sicheren Darmverschluss zu Stande zu bringen. Die Gefahr musste viel grösser sein, als in einem Falle, wo sich eine Darmverwachsung an der Oberfläche der Cyste selbst gefunden hätte; denn wenn wir annehmen, dass sich ein Ovarialtumor um eine Achse dreht, welche die Verlängerung des Stieles bildet, so müssen die Widerstände für eine Drehung wachsen, je näher die Verwachsungen dem Aequator der Cyste liegen. In dem Falle, wo die Darmverwachsung am Aequator liegt, würde einestheils die Cyste eine gewisse Drehung ohne Schaden für den Darm machen können, da ja dieser selbst beweglich an seinem Mesenterium aufgehängt ist und eine gewisse Lageveränderung mitmachen kann; anderentheils könnte gerade am Aequator, wo der grösste Hebelarm wirkt, der gespannte Darm eine begonnene Drehung am leichtesten aufhalten, bezw. durch seine Eigenbewegung rückgängig machen. Allerdings würde bei einer solchen Verwachsung meist 
wohl eine geringere Drehung zur Abknickung des Darmes genügen, als bei einer Verwachsung am Stiele selbst, denn bei einer halben Drehung würde die Ortsveränderung der Anhaftungsstelle im ersteren Falle eine viel bedeutendere sein, als im zweiten Falle. Die Gefahr des Darmverschlusses wäre also für beide Fälle gleich gross, wenn nicht die drehenden Kräfte in dem ersten Falle schon im Beginne der Drehung durch die Verwachsung selbst abgeschwächt würden, während diese am Stiele denselben Kräften fast gar keinen Widerstand entgegensetzt. Damit komme ich auf die drehenden Kräfte selbst zu sprechen, die bereits vielfach erörtert sind, über die wir aber trotzdem keine Klarheit haben. Ohne die Frage hier ausführlich behandeln zu wollen, möchte ich nur einige Erwägungen anknüpfen, auf die ich bei der Betrachtung unseres Falles geführt wurde.

Thorn (a. a. O. S. 49) unterscheidet fünf Ursachen der Achsendrehung: 1) Veränderungen in und am Tumor selbst; 2) physiologische und pathologische Vorgänge in der Becken- und Bauchhöhle und deren Wandungen; 3) Actionen des Körpers in toto und einzelner Theile desselben; 4) Traumen; 5) therapeutische Massnahmen.

Fassen wir zunächst 1) und 5), bei denen zumeist die Verlegung des Schwerpunktes die Drehung bewirkt, zusammen, so kommen diese, wie überhaupt für ausgiebige Drehungen, auch in unserem Falle nicht in Betracht, da es sich um eine glatte einkammerige Cyste handelte. Ebenso war ein Trauma auszuschliessen. Von den unter 2) von Thorn angeführten Einzelursachen könnte nur in Betracht kommen: verschiedener Füllungsgrad der Gedärme, Peristaltik derselben, verschiedener Füllungsgrad der Blase und nicht die übrigen: dazu kommende Schwangerschaft, gleichzeitige Entwicklung eines zweiten Tumor, dazu kommende Geburt, Ascites, regelwidrige Schlaffheit der Bauchdecken, Hängebauch. Dagegen sind von Wichtigkeit die unter 3) angeführten Ursachen: nämlich Bewegungen des Körpers, im besonderen Thätigkeiten, welche mit Steigerung des intraabdominellen Druckes verknüpft sind.

Anamnestisch liess sich nichts nachweisen, was die Ursache zur Stieldrehung hätte sein können, als dass die Frau an dem Tage eine starke Dosis Oleum ricini erhalten hatte und mehrmals zu Stuhle gewesen war. Alle Körperbewegungen wurden von der ängstlichen Frau sehr behutsam ausgeführt, so dass sie nicht direct $\mathrm{zu}$ beschuldigen waren. Es würden also heranzuziehen 
sein: verschiedener Füllungsgrad der Gedärme, Peristaltik, verschiedener Füllungsgrad der Blase und die Bauchpresse bezw. die Stublentleerung. Es ist aber dann wunderbar, dass die Stieldrehung ein so seltenes Ereigniss ist, während alle diese Ursachen bei Ovarialtumoren häufig einwirken. Deshalb muss wohl noch etwas Besonderes die Drehung zu Stande bringen.

Es ist mir aufgefallen, dass man eine Menge von Ursachen angeführt hat, aber dabei die Art der Einwirkung, die Mechanik der Drehung nur sehr wenig oder gar nicht in Betracht gezogen hat. Zunächst liegt es wohl näher, jede Drehung, soweit sie in ihren Folgen in Erscheinung tritt, als eine plötzlich erfolgende aufzufassen, wenn auch nicht geleugnet werden soll, dass sie, so lange sie ohne sichtbare Folgen blieb, sich allmälig entwickelt haben konnte. Doch einerlei, ob plötzlich oder allmälig entstanden, muss die Stieltorsion durch Kräfte verursacht werden, welche auf die Cyste nur von einer Seite her in gleicher Richtung oder von zwei verschiedenen Seiten entgegengesetzt wirken.

Was den Einfluss der peristaltischen Bewegungen des Darmes belangt, so kann dieser nur gering sein, da die Darmbewegung nur eine verhältnissmässig schwache Wirkung haben kann, weil von Reibung zwischen den beiden Serosen des Darmes und der Cyste kaum die Rede sein kann. Wollte man doch schon eine Wirkung aus den streichenden Bewegungen der Darmschlingen ableiten, so müsste man wenigstens annehmen, dass die Bewegung des Darmes an den Berührungspunkten der Cyste annähernd in gleicher Richtung erfolgte. Nähme man an, dass die Darmbewegung mehr stossend durch Antreiben des Darminhaltes wirkte, so müssten auch diese Stösse annähernd in gleicher Richtung wirken, um eine Drehungswirkung zu erzielen. Dabei müssten aber die Darmschlingen so liegen, dass sie entweder von rechts oder von links hinten auf den Tumor einwirken könnten, denn wenn sie gleichmässig vertheilt hinter dem Tumor liegen, so würde der Drehungsanstoss, den der Tumor rechts hinten erhielte, durch den von links hinten her erfolgenden aufgehoben. Es würde also eine Stieldrehung durch die Darmbewegung um so leichter hervorgebracht werden können, je grösser die Peripherie des Tumor wäre, je mehr die Darmschlingen bei ausgiebigem Raume für die Peristaltik auf einer Seite zusammenlägen und je länger und leichter beweglich der Stiel wäre.

Auch die Einwirkung der Bauchpresse, die ja, wenn man von 
der geringen Ungleichmässigkeit der Zwerchfellbewegung absieht, an sich ziemlich gleichmässig von vorn und von oben auf den Tumor wirken muss, kann eine Drehung unmittelbar nicht hervorbringen. Auch hier kann eine Drehung nur zu Staude kommen, wenn Kräfte einseitig von hinten her oder, wenn wir auch die Vorderfläche des Tumor als Angriffspunkt auffassen, diametral entgegengesetzt wirken, also z. B. links hinten und rechts vorn.

Nehmen wir zu der Einwirkung der Bauchpresse noch die der Stuhlentleerung hinzu, so würde also z. B. bei einem rechtsseitigen Ovarialtumor eine Stieldrehung zu Stande kommen können, wenn die Darmschlingen in ihrer Gesammtheit durch das Heruntertreten des Zwerchfelles - Magen und Leber bewegen sich gleichmässig und können ausser Acht gelassen werden - gegen den Tumor von links hinten plötzlich herangedrückt würden. Nun gewinnt der 'Tumor durch die plötzliche Entleerung des Mastdarmes und das Herabsinken der Flexura sigmoidea ins kleine Becken Platz, nach links über die Mittellinie hinüberzutreten, und könnte der vorderen Bauchwand anliegend schon eine rollende Bewegung rechts herum beginnen und die Angriffspunkte links hinten würden vermehrt. Ob die Bauchmuskulatur der rechten Bauchhälfte, der der Tumor anliegt, von rechts und von vorn her auf die Drehung mit einwirken kann, mag dahingestellt bleiben.

Schliesslich wäre der Punkt noch zu berücksichtigen, dass durch die Füllung der Blase und des unteren Darmabschnittes der ganze Tumor höher steht und dass dabei der Stiel straffer gespannt ist. Während bei einer solchen Stellung bei einem einigermaassen breiten Stiele eine Drehung sehr erschwert wäre, wird sie, indem der Tumor beim Entleeren des Darmes und der Blase plötzlich tiefer tritt und der Stiel entspannt wird, sehr erleichtert.

Ob auch ohne die erwähnten Kräfte durch blosse Körperbewegungen eine Drehung der Cyste möglich ist, ist fraglich. Nehmen wir an, es handle sich um einen mittelgrossen rechtsseitigen Ovarialtumor, der eine gewisse Beweglichkeit besitzt, und denken wir uns den Querschnitt des Leibes nierenförmig, so wäre es denkbar, dass beim Einnehmen der Rückenlage der Tumor von der hervorragenden Wirbelsäule her sich rechtsdrehend an der hinteren Bauchhöhlenwand herabrollt. Beim Aufrichten des Körpers würde er dann in gleichem Sinne weiter nach der vorderen Bauchwand rollen. Hierzu wäre aber einmal wieder nöthig, dass 
die Gedärme auf der anderen Seite zusammenlägen, und dann müsste eine gewisse Reibung vorhanden sein, ohne die eine rollende Bewegung nicht möglich ist. Deshalb bleibt die ganze Annahme unwahrscheinlich. Würde dieselbe Bewegung plötzlich und rasch ausgeführt, so würde hauptsächlich wieder die Bauchpresse in Wirkung treten und eine Drehung nur unter den oben angegebenen Bedingungen stattfinden.

Zu der Freund'schen Annahme ${ }^{1}$, dass zu einer Stieldrehung eine primäre Verwachsung des Tumor nöthig sei, will ich nur bemerken, dass, wenn man diese an einem verhältnissmässig festen Punkte, z. B. der Leber, annehmen wollte, sie geradezu eine Erschwerung für die Achsendrehung bilden würde. Es würde sich dann um eine Geschwulst mit zwei Stielen handeln, von denen jeder bei einer Drehung verkürzt werden müsste. Wenn man eine Kugel in ähnlicher Weise zwischen zwei Fäden ausgespannt aufhängte und sie zu drehen versuchte, so würde sie immer wieder die alte Lage einzunehmen suchen, und dies würde noch deutlicher hervortreten, wenn sie wie ein Ovarialtumor an breiten Bändern aufgehängt wäre. Wäre aber der Stiel sehr lang und fände sich die Verwachsung am Darme, d. h. wäre auch dieser zweite Stiel lang, so fiele der Umstand weg, welcher Freund maassgebend war, dass gerade die Aufhängung der Cyste eine besondere Beweglichkeit gewährleisten sollte.

Ich bin mir wohl bewusst, dass ich mich mit diesen Erörterungen in blossen Annahmen bewege, die vielleicht die spätere Erfahrung als falsche kennzeichnen mag, aber gerade die Erfahrung fehlt uns, welche zur Erklärung der Drehung nöthig ist, nämlich: die Beobachtung der Drehungsrichtung, sowie der Lage des 'Tumor und der Darmschlingen zu einander im Bauchraume.

Fall II. Linksseitige Pyosalpinx. Salpingotomie. Ileus. Kothfistel. Heilung.

Frau V., Schuhmachersfrau aus Blomberg, 25 Jahre alt, kam in die Anstalt am 3. October 1889. Früher stets gesund, überstand sie im Sommer 1885 eine leichte Geburt und machte ein normales Wochenbett durch. Danach will ihr aber aufgefallen sein, dass sie bei unvorsichtigen Bewegungen, Stolpern und Aehnlichem einen stechenden Schmerz im Unterleibe empfand. Ein Vierteljahr nach der Niederkunft stellte sich eine grosse Mattig-

1) Tageblatt der Casseler Naturforscher-Versammlung 1878. 
keit und Schläfrigkeit ein, und der Leib war aufgetrieben. Anfangs waren keine, später starke Schmerzen vorhanden, und mit diesen zugleich trat Fieber ein, so dass die Frau ein Halbjahr lang das Bett hüten musste. Im Beginne der Erkrankung litt sie an Durchfällen, später an Verstopfung. Während der ganzen Zeit hat sie eitrigen Ausfluss aus den Geschlechtstheilen gehabt; Eiter im Stuhlgange wurde jedoch nicht bemerkt. Seit vier Jahren konnte die Frau nur gebückt gehen und empfand beim Aufrichten starke Schmerzen, die auch bei ruhigem Stehen immer in gewissem Grade vorhanden waren, schlimmer im liegen, und es kamen noch Kreuzschmerzen hinzu. Es bestand häufiger Stuhldrang, und der Stuhlgang selbst war schmerzhaft. Auch das Harnlassen verursachte ihr einen krampfartigen Schmerz. Seit dem Winter 1888 war ausserdem die Menstruation unregelmässig geworden, häufig von dreiwöchentlicher Dauer und mit starkem Blutverluste verbunden. Vier Wochen vor ihrer Aufnahme hatte sie hohes Fieber und besonders heftige Schmerzen im Leibe.

lhr Leiden machte sie zu jeder häuslichen Arbeit unfähig.

Befund: Kleine Frau, bei ihrer Aufnahme sehr blass und schlecht genährt. Temperatur normal, Puls nie unter 92, häufig 120 in der Minute. Lungen, Herz und Nieren ohne Veränderungen. Unterleibsschmerzen, zum grössten Theile links begrenzt, waren so stark, dass die Frau nur durch Sulfonal Nachts Schlaf fand. Untersuchung in Narkose: In der Scheide ziemlich reichliches, milchiges Secret. Scheidenschleimhaut nicht geröthet, Scheidentheil dick, zeigt starke Röthung in der Umgebung des Muttermundes. Auf Druck gegen den Scheidentheil entleert sich eine eiterähnliche, mit Blut gemischte Flüssigkeit aus dem Cervicalcanale. Vulva blassroth, Bartholinische Drüsen nicht vergrössert, Leistendrüsen nicht geschwollen. Harnröhre ohne Veränderungen. Uterus anteflectirt, etwas vergrössert, hart und mit dem Grunde nach links verzogen. An den Uterus schliesst sich nach links ein etwa wallnussgrosser, empfindlicher Tumor, der als vergrössertes Ovarium angesprochen wird. Von der linken Gebärmutterkante verläuft bogenförmig nach vorn ein etwa fingerdieker geschlängelter derber Strang, der sich bis vor den Uterus nach vorn verfolgen lässt, die linke Tube. Rechts lässt sich das Ovarium nicht deutlich aus einer diffusen Schwellung aussondern, die rechte Tube lässt sich durchfühlen, bildet aber keinen verdickten Strang. Die rechte Douglas'sche Falte erscheint etwas gespannt. 


\section{Diagnose: Linksseitige Salpingitis.}

Therapie: Da die Frau zu jeder Arbeit unfähig geworden ist und andere Behandlungsweisen erfolglos geblieben sind, wird ihr die Laparatomie vorgeschlagen, zu der sie gern ihre Einwilligung giebt. Herr Prof. Runge führte am 19. October die Operation aus. Erhöhte Beckenlagerung. Eröffnung der Bauchhöhle in der Linea alba. Nachdem die Darmschlingen durch Deckschwämme zurückgedrängt sind, liegt die linke Tube in der unteren Schnitthälfte vor.' Sie bildet einen fingerdicken Strang, der halbkreisförmig nach vorn geschlängelt verläuft. Unter ihr schimmert eine dunkelblane Geschwulst hervor, die beim Versuche, sie besser zugänglich und sichtbar zu machen, platzt und ihren blutigen Inhalt in die Banchhöhle ergiesst. Es werden nun Tube und linkes Ovarium entfernt. Als das Ovarium zur Unterbindung des Stieles hervorgezogen wird, platzt in der Tiefe ein Abscess und es quillt gelbflüssiger, stinkender Eiter hervor, der durch Schwämme mit $21 / 2^{-}$ bezw. 5 proc. Carbolsäure sorgfältig aufgetupft wird. Die peritoneale Oberfläche der Blase, sowie des linken Ligamentes sind stark geröthet. Das Netz steht durch eine Verwachsung mit dem parietalen Peritoneum links vom oberen Schnittwinkel in Verbindung. Die Bauchwunde wird durch tiefe, das Peritoneum breit fassende Nähte geschlossen, oberflächliche Zwischennähte werden gelegt, die geschlossene Wunde mit Jodoform gepudert und ein Druckverband angelegt. Die entfernte Tube zeigt sich mit eingedickten Eitermassen erfüllt, ihre Höhlung ist aber eng, während die Wand stark verdickt ist. Eine im hiesigen pathologischen Institute ausgeführte mikroskopische Untersuchung ergab Vermehrung des Bindegewebes und keinerlei Anhaltspunkte für Tuberkulose.

Mittags Temperatur 35:1 Puls 92. Im Laufe des Tages vier Mal Erbrechen schleimig-wässriger Massen. Abends Temperatur 37,3; Puls 110. $15 \mathrm{gtt}$. Tincturae opii simplicis innerlich, dann 0,01 Morphium subcutan, jedoch kein Schlaf in der Nacht.

20. October: Fünf Mal Erbrechen. Schmerzen und geringe Auftreibung des Leibes in der Magengrube mit Angstgef ühl. Erbrochenes hat gallige Färbung und keinen iiblen Geruch. Höchste Temperatur 37,3, höchster Puls 120. 45 gtt. Tincturae opii simplicis im Laufe des Tages.

21. October: Nach schlafloser Nacht bleiben Schmerz in der Magengrube, stete Beängstigung und Erbrechen. Dazu geringe Athemnoth und zeitweises Aufstossen. Am Morgen Verband ge- 
wechselt. Leib etwas aufgetrieben, nicht druckempfindlich. Wundränder nicht geröthet. 90 gtt. Tincturae opii simplicis in sechs Dosen. Höchste Temperatur 37,3; Puls 112.

22. October: In der Nacht wenig Schlaf wegen Schmerzen. Kein Erbrechen mehr. Mittags gehen die ersten Blähungen ab und dadurch etwas Erleichterung. Abends wieder Schmerzen und Beängstigung. Während bisher gegen den Durst nur kleine Eisstückchen und kalter Cognac mit Wasser sehr sparsam. verabreicht waren, erhält die Kranke jetzt Milch theelöffelweise. Abends $20 \mathrm{gtt}$. Tincturae opii simplicis. Höchste Temperatur 37,2; Puls 104.

23. October: Nach einer Opiumgabe, Nachts um 2 Uhr, Schmerzen geringer und etwas Schlaf. Morgens Milch und Bouillon. Im Laufe des Nachmittags steigern sich die Schmerzen, Temperatur erreicht 38,6 bei 130 Pulsen. Dazu Abends 7 Uhr zweimaliges Erbrechen von iibelriechender, nicht gefärbter Flüssigkeit. $100 \mathrm{gtt}$. Opiumtinctur in fünf Dosen. Kleines Klysma von $200 \mathrm{ccm}$ Wasser blieb ohne Wirkung, Schmerzen schienen stärker zu werden.

24. October: Dieselben Schmerzen. Temperatur sinkt bis Abends $10 \mathrm{Uhr}$ allmälig anf 37,8, Puls von 138 auf 132. Der Gedanke an eine Wiedereröffnung der Bauchwunde wird zunächst aufgegeben, da die Temperatur jetzt abgefallen und der Leib nicht aufgetrieben ist. Abends wieder Erbrechen von übelriechender, trüber Flüssigkeit. $100 \mathrm{gtt}$. Tincturae opii simplicis, während des Tages kein Schlaf.

25. October: Nachdem 4 Uhr Morgens $20 \mathrm{gtt}$. Opiumtinctur wegen unerträglicher Schmerzen im ganzen Leibe gegeben waren, tritt um 5 Uhr Erbrechen übelriechender Flüssigkeit ein, und zugleich tritt am Schamberge unter dem Verbande dünnflüssiger, dunkelbrauner Koth hervor. Beim Oeffnen des Verbandes zeigt sich, dass der Koth aus einer Oeffnung im unteren Wundwinkel, deren Ränder geröthet sind, mit Blasen von Darmgasen gemischt, stetig fliessend hervorquillt. Auf der linken Seite fühit man in der Höhe dieser Oeffnung eine feste Stelle. Die Schnittwunde oberhalb der Fistel wird dick mit Jodoform bestreut und ein neuer Verband so angelegt, dass nur die Fistelöffnung frei bleibt. Zuerst wird der reichlich fliessende Koth durch zwei Wärterinnen beständig mit Watte entfernt, bis bei Tagesanbruch die Aussonderung spärlicher wird. Dann werden Gazecompressen aufgelegt, die mit 1 proc. 
vor und nach der Laparatomie, beide mit Ausgang in Heilung.

Kreolinlösung getränkt sind, und von Zeit zu Zeit erneuert. Im Laufe des Tages $50 \mathrm{gtt}$. Opiumtinctur. Die Schmerzen haben aufgehört. Temperatur 37,5-37,3, Abends 37,8; Puls 124-120, Abends 128.

26. October: Nachts hat die Frau einige Stunden mit Unterbrechungen geschlafen. Kothaussonderung geringer, wird heller und zuweilen mit Streifen von Eiter. Temperatur Morgens 37,6, Nachmittags 38,6, Abends 38,3; Puls entsprechend 120, 128, 120. Die Frau wird auf die rechte Seite gelagert, um das Abfliessen des Kothes zu erleichtern. Klysma von $300 \mathrm{ccm}$ ohne Wirkung. 70 gtt. Opiumtinctur in vier Gaben.

27. October: Hat gut geschlafen. Absonderung der Kothfistel mehr eiterähnlich, doch Kothgeruch. Temperatur Morgens 38,3, Mittags 38,5, Abends 38,0; Puls 128. 35 gtt. Opiumtinctur.

28. October: In der Nacht neue Schmerzen im Leibe, 25 gtt. Opiumtinctur. Die Fistel liefert keinen Koth mehr, nur noch gelben geruchlosen Eiter. Am Morgen Blähungen. Oleum ricini bewirkt drei Mal Stuhlgang auf natürlichem Wege. Temperatur Morgens 37,9, Mittags 37,6, Abends 38,2; Puls 124-116.

Von diesem Tage an wird die Temperatur normal, die Kranke befindet sich wohl und erholt sich rasch. Die Fistelöffnung bestand $\mathrm{zu}$ der Zeit aus einer haselnussgrossen Grube mit granulirendem Grunde, aus dem auf Druck von links her Eiter austritt. Die Grube verstrich allmälig, die Fistel bestand jedoch noch am 15. December, als die Frau auf ihren Wunsch entlassen wurde. Zur Linken von der Fistel bestand die oben erwähnte, etwa apfelgrosse feste Stelle, welche auch von der Scheide her fühlbar war. Auf Druck entleert sich noch immer Eiter, der aber sonst so spärlich fliesst, dass der Verband nur jeden zweiten Tag gewechselt zu werden braucht. - Am. 10. December, dem 53. Tage nach der Operation, wurde aus der Fistelöffnung ein lose liegender Seidenfaden herausgezogen, von dem es fraglich war, ob er den Bauchdeckennähten angehörte oder aus der Bauchhöhle stammte. Das Aussehen der Frau war bei ihrer Entlassung ge radezu blühend, der Gang leicht und gerade, Sohmer$z \in n$ bestanden nicht mehr. Anfangs Januar schrieb die Frau, dass sie seit acht Tagen sich mehrfach geknüpfte Seidenfäden aus der Fistelöffnung gezogen habe und legte auch davon vier Stück dem Briefe bei. Es waren doppeltgeknotete Seidennähte, die 
uffenbar dem versenkten Stiele angehört hatten. Während dieser "Leit will sie Schmerzen in der linken Seite, heftige Kreuzschmerzen und beim Stuhlgange Schmerzen im Mastdarme empfunden haben.

Als am 1. Februar dieses Jahres die Frau sich wieder vorstellte, war ihr Aussehen vortrefflich. In die Fistel, welche noch immer aussonderte, drang eine Uterussonde nach links abweichend bis zur Knickung leicht ein. Die feste Stelle auf der linken Seite war kaum noch zu fühlen, wohl aber von der Scheide wie vom Mastdarme aus leicht eine feste Stelle auf der rechten Seite, welche druckempfindlich war. Von selbst auftretende links lokalisirte Schmerzen werden durch warme Bäder bald gehoben. Nur beim Stuhlgange bleibt geringer Schmerz. Die Frau ging dann in ihre Heimath, um die Bäderbehandlung fortzusetzen. Anfang Juni war die Fistel etwa eine Woche lang geschlossen, öffnete sich aber wieder und sonderte mit dem Eintritte der Regel Blut aus, was auch im April schon einmal vorgekommen war. Lin Zusammenhang zwischen Gebärmutter und Fistelgang bestand nicht.

Von den sechs in der Literatur bekannten Fällen von Darmfisteln nach Laparatomie ${ }^{1}$ ) sind die Fälle von $\mathrm{Keith}^{2}$ ) und von Bryant ${ }^{3}$ unserem Falle am ähnlichsten. Im. Falle Keith bildete sich nach sechs Wochen ein Beckenabscess, der durchbrach und später erst sich in eine Kothfistel umwandelte. Im Falle Bryant öffnete sich im unteren Wundwinkel am 17. Tage ein Abscess, der sich nach vier Tagen schloss und dann sich nach einer Woche als Kothfistel wieder öffnete. Auch hier wurden Fäden ausgestossen, dann schloss sich die Wunde von selbst und dauernd.

In unserem Falle liegt die Ursache der Kothfistel sehr klar. Es bildete sich entweder von dem versenkten Reste der Tuben her oder von dem in der Nähe gelegenen geborstenen Abscesse durch zurückgebliebene Infectionskeime oder, was weniger wahrscheinlich, durch Verunreinigungen, welche durch die Seide oder mit der Hand hineingetragen wurden, ein gegen die Bauchböhle abgekapseltes Exsudat, welches allmälig an Grösse zunahm. An-

1) Hegar und Kaltenbach, Operative Gynäkologie, 3. Aufl, S. 312. Olshausen, Ovarien, S. 381.

2) Glasgow Medical Journal 1867, p. 295.

3) Lancet, 1. Februar 1868. 
vor und nach der Laparatomie, beide mit Ausgang in Heilung. 305

fangs wurde der Darm dadurch so wenig berührt, dass am vierten Tage Blähungen abgehen konnten, dann aber drängte es stark auf eine Darmschlinge und entwickelte dadurch alle Erscheinungen des lleus. Jedenfalls zerstörte es zuerst die Darmwand, so dass sich nun der Darminhalt in den Abscess ergoss. Zugleich oder infolge des Kothergusses vergrösserte sich aber der Abscess auch nach der Bauchwand hin und brach schliesslich im unteren Wundwinkel als Kothfistel durch. Mit diesem Durchbruche hörte aber der Druck auf die Darmschlinge auf, der natürliche Weg wurde für den Koth wieder frei und die eigentliche Darmfistel heilte. 